\title{
The Atacama Desert: A Biodiversity Hotspot and Not Just a Mineral-Rich Region
}

\author{
Benito Gómez-Silva ${ }^{1 *}$ and Ramón Alberto Batista-García ${ }^{2}$ \\ ${ }^{1}$ Laboratory of Biochemistry, Biomedical Department, Health Sciences Faculty and Centre for Biotechnology and \\ Bioengineering (CeBiB), Universidad de Antofagasta, Antofagasta, Chile, ${ }^{2}$ Centro de Investigación en Dinámica Celular, \\ Instituto de Investigación en Ciencias Básicas y Aplicadas, Universidad Autónoma del Estado de Morelos, Cuernavaca, \\ Mexico
}

Keywords: The Atacama, biodiversity, extreme environments, extremophiles, lithobionts, new paradigm

\section{INTRODUCTION}

The Atacama Desert in northern Chile is a coastal nonpolar hyperarid desert with nearly $1,000 \mathrm{~km}$ long located in South America (latitudes $19^{\circ} \mathrm{S}$ and $30^{\circ} \mathrm{S}$ ), between the Pacific Ocean to the west and the Andes Range to the east (Bull et al., 2016). It is also considered the oldest and driest desert on Earth (Houston and Hartley, 2003; McKay et al., 2003; Sun et al., 2010). Historically, the Atacama Desert has been described as a barren, desolate, lifeless, harsh environment for life with an undisputed mineral richness under exploitation since pre-Columbian times (Philippi, 1860; OPSAL, 2021). A solid and growing body of information on microbial and genetic richness of the Atacama Desert has been published during the first two decades of the twenty-first century. It sustains that this hyperarid region is no longer just a mineral-rich and sterile territory and must be conceptually redefined to include its biological resources. This integral view of the Atacama

Edited by:

Virginia Helena Albarracín, CONICET Center for Electron Microscopy (CIME), Argentina

Reviewed by:

Juan Castro-Severyn, Catholic University of the North, Chile

*Correspondence: Benito Gómez-Silva benito.gomez@uantof.cl

Specialty section: This article was submitted to Extreme Microbiology, a section of the journal

Frontiers in Microbiology

Received: 11 November 2021 Accepted: 04 January 2022 Published: 09 February 2022

Citation: Gómez-Silva B and Batista-García RA (2022) The Atacama Desert: A Biodiversity Hotspot and Not Just a Mineral-Rich Region. Front. Microbiol. 13:812842. doi: 10.3389/fmicb.2022.812842
Desert ecosystem should raise environmental, social, and educational impacts as well as scientific and technological progress in the region. The reports cited in this contribution are a selection from a large body of substantial and informative articles on the microbiology of the Atacama.

\section{BASES FOR A NEW VIEW OF THE ATACAMA DESERT}

Articles published by the mid-1960s and at the beginning of the present century (Cameron et al., 1966; Dose et al., 2001; McKay et al., 2003; Navarro-González et al., 2003) have been considered seminal and a driving impulse for a substantial number of microbiology-related reports on the Atacama Desert. Figure 1 provides a comprehensive and quantitative overview on the number and types of scientific publications on different disciplines on the Atacama since 1972, particularly on ecology, genetics, and microbiological studies conducted during the present century.

Life in our planet proliferates in almost any habitat known to have available liquid water sources. Abundance and diversity of life forms are limited by the prevailing physical and chemical variables in the Atacama Desert, as well as in other extreme environments on Earth (Rothschild and Mancinelli, 2001; Gómez-Silva, 2010; Bull et al., 2016; Meslier et al., 2018). High desiccation (aridity index near or below 0.05) and one of the highest solar insolation in our planet (UV index of 15-20) are the two major environmental factors limiting life in the Atacama and highly restrictive to microorganisms without the appropriate strategies to cope with them (Houston and Hartley, 2003; McKay et al., 2003; Cordero et al., 2014; Bull et al., 2016; Gómez-Silva, 2018; Meslier et al., 2018). Not surprisingly, the Atacama has a relatively abundant microbial population at wetter habitats such as wetlands and nonfossil salars (salt flats with surface and/or underground liquid water inflows from rains and rivers, e.g., Salar de Atacama and Salar de Llamara). Many articles have shown the 
A

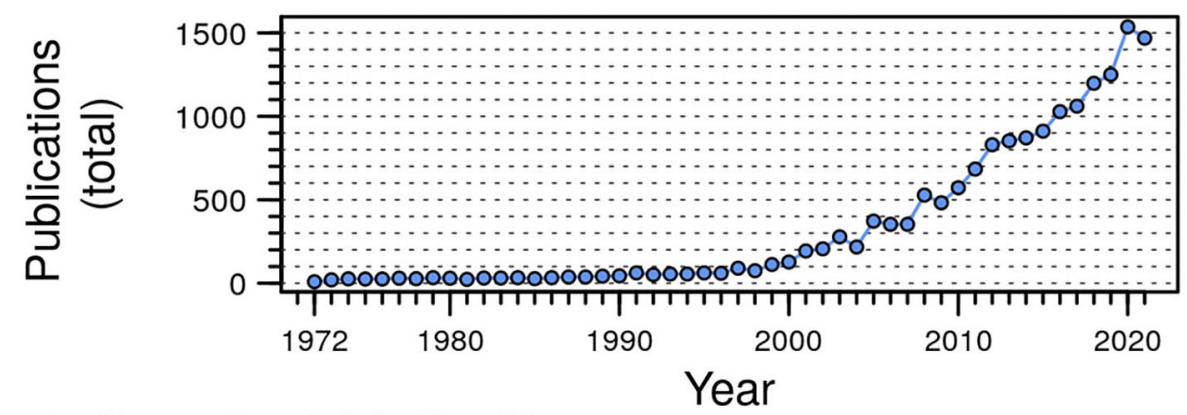

B

Atacama Desert - Scientific articles

Atacama Desert - Scientific articles (total)

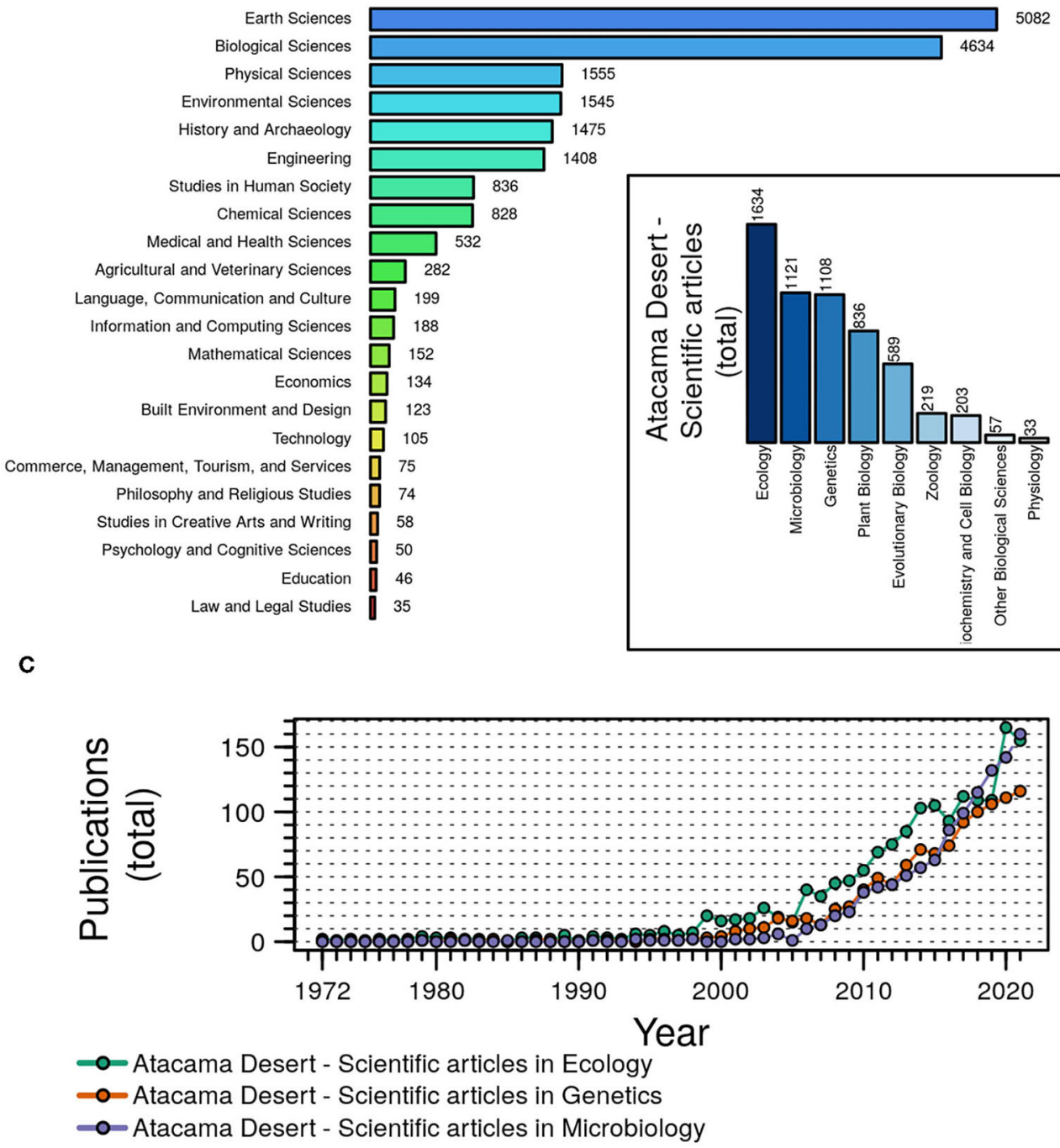

FIGURE 1 | Dynamic of the scientific article production about the Atacama Desert in the period 1972-2021. (A) Publication of scientific articles about the Atacama Desert by year. (B) Distribution of the number of scientific publications about the Atacama Desert in different research categories. The inserted panel shows the number of publications in different subcategories belonging to biological sciences. (C) Scientific publication on the Atacama Desert by year in three different subcategories: Ecology, Microbiology, and Genetics. Figure was prepared in R environment (R base). Data of publications was collected from https://app.dimensions. ai on November 07, 2021. 
experience of research groups in isolation, culturing, taxonomy, metabolic capabilities, genomic studies and, biochemical characterization of individual microorganisms and microbial consortia from the Atacama (Cabrol et al., 2007; Rainey et al., 2007; Dorador et al., 2010; Gramain et al., 2011; Farías et al., 2014; Bull et al., 2016; Finstad et al., 2017; Castro et al., 2018; Santiago et al., 2018; Warren-Rhodes et al., 2019; Flores et al., 2020; Galetovic et al., 2020; Salazar-Ardiles et al., 2020; Shen et al., 2021a; Vignale et al., 2021; Villalobos et al., 2021; among many other important reports). Microbial research has also been focused on the Atacama's habitats without any regular source of liquid water, such as soils, rocks, and fossil salars. Lithic substrates are proper refuges for microbial colonization in the absence of regular supply of liquid water, a fact with evident astrobiological implications (Wierzchos et al., 2006; Gómez-Silva, 2010, 2018; Gramain et al., 2011; Robinson et al., 2015; Finstad et al., 2017; Meslier et al., 2018). Extensive metagenomics studies have demonstrated that lithobiontic life in the Atacama includes members of the three domains of life and viruses (Gómez-Silva et al., 2019; Uritskiy et al., 2019; Hwang et al., 2021). Hipolithic, endolithic, or epilithic colonization by cyanobacteria-dominated microbial consortia have been reported in quartz, halites and gypsum substrates, where the microbial communities harvest liquid water from atmospheric water vapor by salt deliquescence (Davila et al., 2008), capillary condensation (Wierzchos et al., 2012), and fog droplets (Azúa-Bustos et al., 2011).

Bioprospection and analyses of microbiological, metagenomics and other studies on novel extremophiles and extreme-tolerant microorganisms from the Atacama have opened new biotechnological opportunities based on the search of novel secondary metabolites, genes, gene clusters, metabolic pathways, peptides, pigments, and macromolecules, with impacts in biomedicine, food and feed supplements, biological control and other activities (Rateb et al., 2011; Gonçalves et al., 2015; Gómez-Silva et al., 2019; Flores et al., 2020; Galetovic et al., 2020; Salazar-Ardiles et al., 2020).

Twenty years of active research in the Atacama have provided substantial microbiological information; however, additional studies are required to properly assess the role of the microbial community structure in ecosystem functioning, provisioning, and supporting services. Chemolithotroph life in the Atacama is an example of pending issues to be addressed in future studies focused on its ecological value. Metagenomics studies on the halite microbiome in the Atacama have shown a limited genetic repository for sulfate oxidation but the presence of genes of all the enzymes involved in the assimilatory sulfate reduction pathway, with direct implication on sulfate uptake from oceanic fog, amino acid biosynthesis, microbial metabolism, and sulfur cycle (Gómez-Silva, 2010; Gómez-Silva et al., 2019). Also, active metabolic capabilities such as photosynthetic and transcriptional activities have been demonstrated in microbial consortia inhabiting halites and wetlands, and nitrogen cycling along the rainfall gradient in the Atacama (Davila et al., 2015; Orellana et al., 2020; Uritskiy et al., 2020; Castro-Severyn et al., 2021; Shen et al., 2021b). New questions and ingenious experimental approaches are needed to produce comprehensive knowledge about the role of microbes in biogeochemical cycles, the cellular bases that assist the crosstalk between microbial cells, the trans-kingdom molecular mechanisms playing key roles in the gene expression dynamic, among other topics not covered to date. Undoubtedly, the answers to these and other questions will decisively contribute to the understanding of the ecological role of microbial life in the Atacama.

Although not commented in depth in this document, the Atacama Desert harbors a unique vegetation with many endemic lineages and endangered species, mostly restricted to coastal fog oases or lomas formations and at pre-Andes and Andes ranges. These members of the Atacama's biodiversity have adapted their life cycles to selective sites along this hyperdesert and, locations and identification of Cactaceae and other vascular plants in the Atacama have been acknowledged in molecular and botanical reports but also by an ancestral savvy (Rundel et al., 1991; Ruhm et al., 2020; Eshel et al., 2021). The Atacama plants are essential elements of the Atacama scenery and its natural beauty but also for biochemical research and biotechnological applications. Regulations against illegal sampling of plants, seeds and microbes for scientific and commercial purposes are urgently needed.

\section{DISCUSSION}

The Atacama Desert is a lifeless territory, worth only for the exploitation of its mineral content. This summary notion, still present worldwide, supports massive extractive activities with evident and assessable environmental damages, minimal positive cultural impacts but ethno-cultural fractures in the local communities. Centuries-old natural hydrogeological processes have generated underground and surface water bodies on the Salar de Atacama, a pre-Andean endorheic basin with an impressive diversity of life forms adapted to salinity, high solar radiation, and daily and seasonal temperature changes, with important nesting and feeding sites. Also, underground brines with high salts content (lithium and others) are today pumped to evaporation ponds with serious impact on the water content of aquifers and on biodiversity (OPSAL, 2021). It is interesting to contrast this limited conception of the Atacama with the ethnographic and anthropological evidence that trace human settlements in the Atacama territory back to the end of the Pleistocene to the present days. The inherited ancestral knowledge has allowed generations of the Atacama's inhabitants to live in close communion with the biological resources of this extreme region, in a respectful and intelligent practice, particularly with the underground and surface water sources (Philippi, 1860; Núñez et al., 2010; Babidge, 2015; Rivera et al., 2018).

Based on the Atacama biological richness, the proposal of an alternative and conceptually broader vision of this extreme dryland is upheld today by activities associated to the use of renewable energy sources (solar and wind), seawater desalination, and tourism for special interests (mountains, lakes, ponds, vegetation, and wetlands). The Atacama is also a natural laboratory that has and will provide solid advances in paleontology, archaeology, anthropology, astronomy, astrobiology, and other disciplines. It seems evident and 
necessary that a "new Atacama Desert" be recognized as a desert biome containing mineral richness, highly diverse extant life forms, renewable energy resources, geographic, oceanic, and astronomical assets, and inhabitants with an inherited ancestral acknowledge. New commercial ventures on its natural resources must be carefully regulated to avoid, not only diminishing, their environmental impacts. The biological resources of the Atacama biome, as well as the effects on living organisms and human communities, have not and are not properly protected today by the Chilean legislation; more information can be obtained from documents associated to the Nagoya Treaty (https://www.cbd. int/countries/?country=cl), Babidge (2015) and OPSAL (2021). The intrinsic value of the Atacama biodiversity needs urgent management and protection by proper and pending regulatory policies. Members of the microbial life inhabiting sites with commercially extractive interests in the Atacama (salars, land, mountains, and water sources) are particularly endangered since "they cannot be seen" as easily as plants and animals. Today, the microbiome colonizing extreme habitats in the Atacama Desert is invaluable evidence on the evolution of life in our planet; they might not have "commercial" value but represent scientific and cultural assets subjected to irreversible loss.

Social and natural sciences are contributing to a better understanding of this hyperarid land and the universe around us.

\section{REFERENCES}

Azúa-Bustos, A., González-Silva, C., Mancilla, R. A., Salas, L., Gómez-Silva, B., McKay, C. P., et al. (2011). Hypolithic cyanobacteria supported mainly by fog in the coastal range of the Atacama Desert. Microb. Ecol. 61:568-581. doi: 10.1007/s00248-010-9784-5

Babidge, S. (2015). Contested value and an ethics of resources: water, mining and indigenous people in the Atacama Desert, Chile. Aust. J. Anthropol. 27, 84-103. doi: $10.1111 /$ taja.12139

Bull, A. T., Asenjo, J. A., Goodfellow, M., and Gómez-Silva, B. (2016). The Atacama desert: technical resources and the growing importance of novel microbial diversity. Annu. Rev. Microbiol. 70, 215-234. doi: 10.1146/annurev-micro-102215-095236

Cabrol, N. A., Wettergreen, D., Warren-Rhodes, K., Grin, E. A., Moersch, J., Chong Diaz, G., et al. (2007). Life in the Atacama: searching for life with rovers (science overview). J. Geophys. Res. 112:G04SG02, doi: 10.1029/2006JG000298

Cameron, R. E., Gensel, D. R., and Blank, C. B. (1966). "Soil studies-desert microflora. XII. Abundance of microflora in soil samples from the Chile Atacama Desert," in Supporting Research and Advanced Developments, Space Programs Summary No. 37-38, Vol. IV (Pasadena, CA: Jet Propulsion Lab, NASA), 140-147.

Castro, J. F., Nouioui, I., Sangal, V., et al. (2018). Geodermatophilus chilensis sp nov., from soil of the Yungay core-region of the Atacama Desert, Chile. Syst. Appl. Microbiol. 41, 427-436. doi: 10.1016/j.syapm.2018.03.005

Castro-Severyn, J., Pardo-Esté, C., Mendez, K. N., Fortt, J., Marquez, S., Molina, F., et al. (2021). Living to the high extreme: unraveling the composition, structure, and functional insights of bacterial communities thriving in the arsenic-rich Salar de Huasco altiplanic ecosystem. Microbiol. Spectr. 9, e00444-e00421. doi: 10.1128/Spectrum.00444-21

Cordero, R. R., Seckmeyer, G., Damiani, A., Riechelmann, S., Rayas, J., Labbe, F., et al. (2014). The world's highest levels of surface UV. Photochem. Photobiol. Sci. 13, 70-81. doi: 10.1039/C3PP50221J

Davila, A. F., Gómez-Silva, B., De Los Ríos, A., Ascaso, C., Olivares, H., McKay, C. P., et al. (2008). Facilitation of endolithic microbial survival in the hyperarid core of the Atacama Desert by mineral deliquescence. J. Geophys. Res. 113:G01028. doi: 10.1029/2007JG000561
The Atacama Desert is no longer the same has we have previously learned to know. It would be expected that this new perspective of "a new Atacama Desert," if broadly accepted, be included in educational plans for new generations of children, researchers, and be shared with the not-scientific society, in Chile and abroad.

\section{AUTHOR CONTRIBUTIONS}

BG-S and RB-G were responsible of all the content expressed in this Opinion article. All authors contributed to the article and approved the submitted version.

\section{FUNDING}

This work was supported by CONICYT grant CeBiB FB-0001 (Chile) and CONACYT grant 1559 (Mexico).

\section{ACKNOWLEDGMENTS}

We acknowledge the support given by our institutions and funding agencies. Authors appreciate very much the contribution of Yordanis Pérez-Llano and Tonatiuh Moreno-Perlín in the preparation of Figure 1.

Davila, A. F., Hawes, I., Araya, J. G., Gelsinger, D. R., DiRuggiero, J., Ascaso, C., et al. (2015). In situ metabolism in halite endolithic microbial communities of the hyperarid Atacama Desert. Front. Microbiol. 6:1035. doi: 10.3389/fmicb.2015.01035

Dorador, C., Vila, I., Remonsellez, F., Imhoff, J. F., and Witzel, K.-P. (2010). Unique clusters of Archaea in Salar de Huasco, an athalassohaline evaporitic basin of the Chilean Altiplano. FEMS Microbiol. Ecol. 73, 291-302. doi: 10.1111/j.1574-6941.2010.00891.x

Dose, K., Bieger-Dose, A., Ernst, B., Feister, U., Gómez-Silva, B., Klein, A., et al. (2001). Survival of microorganisms under the extreme conditions of the Atacama Desert. Origins Life Evol. Biosphere 31, 287-303, 2001. doi: 10.1023/A:1010788829265

Eshel, G., Araus, V., Undurraga, S., Soto, D. C., Moraga, C., Montecinos, A., et al. (2021). Plant ecological genomics at the limits of life in the Atacama Desert. Proc. Natl. Acad. Sci. U. S. A. 118:e2101177118. doi: 10.1073/pnas.2101177118

Farías, M. N., Contreras, M., Rasuk, M. C., Kurth, D., Flores, M. R., Poiré, D. G., et al. (2014). Characterization of bacterial diversity associated with microbial mats, gypsum evaporites and carbonate microbialites in thalassic wetlands: Tebenquiche and La Brava, Salar de Atacama, Chile. Extremophiles 18, 311-329. doi: 10.1007/s00792-013-0617-6

Finstad, K. M., Probst, A. J., Thomas, B. C., Andersen, G. L., Demergasso, C., Echeverría, A., et al. (2017). Microbial community structure and the persistence of cyanobacterial populations in salt crusts of the hyperarid Atacama desert from genome-resolved metagenomics. Front. Microbiol. 8:1435. doi: 10.3389/fmicb.2017.01435

Flores, N., Hoyos, S., Venegas, M., Galetovic, A., Zuñiga, L. M., Fábrega, F., et al. (2020). Haloterrigena sp. strain SGH1, a bacterioruberin-rich, perchloratetolerant halophilic archaeon isolated from halite microbial communities, Atacama Desert, Chile. Front. Microbiol. 11:324. doi: 10.3389/fmicb.2020.00324

Galetovic, A., Seura, F., Gallardo, V., Graves, R., Cortés, J., Valdivia, C., et al. (2020). Use of phycobiliproteins from Atacama cyanobacteria as food colorants in a dairy beverage prototype. Foods 99:244. doi: 10.3390/foods9020244

Gómez-Silva, B. (2010). "On the limits imposed to life by the hyperarid Atacama desert in Northern Chile," in Astrobiology: Emergence, Search and Detection of Life. Chapter 9, ed V. A. Basiuk (Los Angeles, CA: American Scientific Publishers), 199-213. 
Gómez-Silva, B. (2018). Lithobiontic life: Atacama rocks are well and alive. Antoine van Leeuwenhoek 111, 1333-1345. doi: 10.1007/s10482-018-1033-9

Gómez-Silva, B., Vilo-Muñoz, C., Galetovic, A., Dong, Q., CastelánSánchez, H. G., Pérez-Llano, Y., et al. (2019). Metagenomics of Atacama lithobiontic extremophile life unveils highlights on fungal communities, biogeochemical cycles, and carbohydrate-active enzymes. Microorganisms 7:619. doi: 10.3390/microorganisms7120619

Gonçalves, V. N., Cantrell, C. L., Wedge, D. E., Ferreira, F. C., Soares, M. A., Jacob, M. R., et al. (2015). Fungi associated with rocks of the Atacama Desert: taxonomy, distribution, diversity, ecology and bioprospection for bioactive compounds. Environ. Microbiol. 23, 4200-13. doi: 10.1111/1462-2920.13005

Gramain, A., Chong Díaz, G., Demergasso, C., Lowenstein, T. K., and McGenity, T. J. (2011). Archaeal diversity along a subterranean salt core from the Salar Grande. Environ. Microbiol. 13, 2105-2021. doi: 10.1111/j.1462-2920.2011.02435.x

Houston, J., and Hartley, A. J. (2003). The central Andean west-slope rainshadow and its potential contribution to the origin of hyper-aridity in the Atacama Desert. Int. J. Climatol. 23, 1453-1464. doi: 10.1002/joc.938

Hwang, Y., Rahlff, J., Schulze-Makuch, D., Schloter, M., and Probst, A. J. (2021). Diverse viruses carrying genes for microbial extremotolerance in the Atacama Desert hyperarid soil. mSystems 6, e00385-e00321. doi: 10.1128/mSystems.00385-21

McKay, C. P., Friedmann, E. I., Gómez-Silva, B., Cáceres-Villanueva, L., Andersen, D. L., and Landheim, R. (2003). Temperature and moisture conditions for life in the extreme arid region of the Atacama Desert: four years of observations including the El Nino of 1997-1998. Astrobiology 3, 393-403. doi: $10.1089 / 153110703769016460$

Meslier, V., Casero, M. C., Dailey, M., Wierzchos, J., Ascaso, C., Artieda, O., et al. (2018). Fundamental drivers for endolithic microbial community assemblies in the hyperarid Atacama Desert. Environ. Microbiol. 20, 1765-1781. doi: 10.1111/1462-2920.14106

Navarro-González, R., Rainey, F. A., Molina, P., Bagaley, D. R., Hollen, B. J., de la Rosa, J., et al. (2003). Mars-like soils in the Atacama Desert, Chile, and the dry limit to microbial life. Science 302, 16089-16094. doi: 10.1126/science. 1089143

Núñez, L., Grosjean, M., and Cartajena, J. (2010). Sequential analysis of human occupation patterns and resource use in the Atacama Desert. Chungará 42, 363-391. doi: 10.4067/S0717-73562010000200003

OPSAL (2021). Salares Andinos: Ecología de Saberes por la Protección de nuestros Salares y Humedales. Available online at: https://cl.boell.org/es/2020/12/18/ salares-andinos-ecologia-de-saberes-por-la- proteccion-de-nuestros-salaresy-humedales (accessed October 17, 2021).

Orellana, G., Gómez-Silva, B., Urrutia, M., and Galetovic, A. (2020). UV-A irradiation increases scytonemin biosynthesis in cyanobacteria inhabiting halites at salar grande, Atacama desert. Microorganisms 8:1690. doi: 10.3390/microorganisms 8111690

Philippi, R. A. (1860). Viage al Desierto de Atacama: hecho de orden del gobierno de Chile en el verano 1853-54. Halle en Sajonia: Librería de Eduardo Anton. Available online at: http://www.memoriachilena.gob.cl/602/w3-article-7825. html (accessed September 30, 2021).

Rainey, F. A., Ferreira, M., Nobre, M. F., Ray, K., Bagaley, D., Earl, A. M., et al. (2007). Deinococcus peraridilitoris sp. nov., isolated from a coastal desert. Int. J. Syst. Evol. Microbiol. 57, 1408-1412.

Rateb, M. E., Houssen, W. E., Arnold, M., Abdelrahman, M. H., Deng, H., Harrison, W. T. A., et al. (2011). Chaxamycins A-D, bioactive ansamycins from a hyper-arid desert Streptomyces sp. J. Nat. Prod. 74, 1491-1799. doi: $10.1021 / \mathrm{np} 200320 \mathrm{u}$

Rivera,. M., Galetovic, A., Licuime, R., and Gómez-Silva, B. (2018). A microethnographic and ethnobotanical approach to Llayta consumption among the Andes feeding practices. Foods 7:202. doi: 10.3390/foods7120202

Robinson, C. K., Wierzchos, J., Black, C., Crist-Christoph, A., Ma, B., Ravel, J., et al. (2015). Microbial diversity and the presence of algae in halite endolithic communities are correlated to atmospheric moisture in the hyper-arid zone of the Atacama Desert. Environ. Microbiol. 1, 299-315. doi: $10.1111 / 1462-2920.12364$

Rothschild, L., and Mancinelli, R. (2001). Life in extreme environments. Nature 409, 1092-1101. doi: 10.1038/35059215

Ruhm, J., Böhnert, T., Weigend, M., Merklinger, F. F., Stoll, A., Quandt, D., et al. (2020). Plant life at the dry limit - spatial patterns of floristic diversity and composition around the hyperarid core of the Atacama Desert. PLoS ONE 15:e0233729. doi: 10.1371/journal.pone.0233729

Rundel, P. W., Dillon, M. O., Palma, B., Mooney, H. A., and Gulmon, S. L. (1991). The phytogeography and ecology of the coastal atacama and peruvian deserts. Aliso J. Systemat. Evol. Bot. 13, 1-49. doi: 10.5642/aliso.19911301.02

Salazar-Ardiles, C., Caimanque, T., Galetović, A., Vilo, C., Araya, J. E., Flores, N., et al. (2020). Staphylococcus sciuri strain LCHXa is a free-living lithiumtolerant bacterium isolated from Salar de Atacama, Chile. Microorganisms 8:668. doi: 10.3390/microorganisms 8050668

Santiago, I. F., Gonçalves, V. N., Gómez-Silva, B., Galetovic, A., and Rosa, L. H. (2018). Fungal diversity in the Atacama Desert. Antonie van Leeuwenhoek 111, 1345-1360. doi: 10.1007/s10482-018-1060-6

Shen, J., Wyness, A. J., Claire, M. W., and Zerkle, A. L. (2021a). Spatial variability of microbial communities and salt distributions across a latitudinal aridity gradient in the Atacama desert. Microb. Ecol. 82, 442-458. doi: $10.1007 /$ s00248-020-01672-w

Shen, J., Zerkle, A. L., and Claire, M. W. (2021b). Nitrogen cycling and biosignatures in a hyperarid mars analog environment. Astrobiology 22:12. doi: 10.1089/ast.2021.0012

Sun, T., Bao, H., Reich, M., and Hemming, S. R. (2010). More than ten million years of hyper-aridity recorded in the Atacama Gravels. Geochim. Cosmochim. Acta 227, 123-132. doi: 10.1016/j.gca.2018.02.021

Uritskiy, G., Getsin, S., Munn, A., Gomez-silva, B., Davila, A., Glass, B., et al. (2019). Halophilic microbial community compositional shift after a rare rainfall in the Atacama Desert. ISME J. 13, 2737-2749. doi: 10.1038/s41396-0190468-y

Uritskiy, G., Tisza, M. J., Gelsinger, D. G., Munn, A., Taylor, J., and DiRuggiero, J. (2020). Cellular life from the three domains and viruses are transcriptionally active in a hypersaline desert community. Environ. Microbiol. 23, 3401-3417. doi: $10.1111 / 1462-2920.15023$

Vignale, F. A., Kurth, K., Lencina, A. I., Poiré, D. G., Chihuailaf, E., Muñoz-Herrera, N. C., et al. (2021). Geobiology of andean microbial ecosystems discovered in Salar de Atacama, Chile. Front. Microbiol. 12:762076. doi: $10.3389 /$ fmicb. 2021.762076

Villalobos, A. S., Wiese, J., Borchert, E., Rahn, T., Slaby, B. M., Steiner, L. X., et al. (2021). Micromonospora tarapacensis sp. nov., a bacterium isolated from a hypersaline lake. I. J. Syst. Evol. Microbiol. 71:5109. doi: 10.1099/ijsem.0. 005109

Warren-Rhodes, K. A., Lee, K. C., Archer, C. D. J., Cabrol, N., NgBoyle, L., Wettergreen, D., et al. (2019). Subsurface microbial habitats in an extreme desert mars-analog environment. Front. Microbiol. 10:69. doi: $10.3389 /$ fmicb.2019.02129

Wierzchos, J., Ascaso, C., and McKay, C. P. (2006). Endolithic cyanobacteria in halite rocks from the hyperarid core of the Atacama desert. Astrobiology 6, 415-422. doi: 10.1089/ast.2006.6.415

Wierzchos, J., Dávila, A. F., Sánchez-Almazo, I. M., Hajnos, M., Swieboda, R., and Ascaso, C. (2012). Novel water source for endolithic life in the hyperarid core of the Atacama Desert. Biogeosciences 9, 2275-2286. doi: 10.5194/bg-9-2275-2012

Conflict of Interest: The authors declare that the research was conducted in the absence of any commercial or financial relationships that could be construed as a potential conflict of interest.

Publisher's Note: All claims expressed in this article are solely those of the authors and do not necessarily represent those of their affiliated organizations, or those of the publisher, the editors and the reviewers. Any product that may be evaluated in this article, or claim that may be made by its manufacturer, is not guaranteed or endorsed by the publisher.

Copyright $\odot 2022$ Gómez-Silva and Batista-García. This is an open-access article distributed under the terms of the Creative Commons Attribution License (CC BY). The use, distribution or reproduction in other forums is permitted, provided the original author(s) and the copyright owner(s) are credited and that the original publication in this journal is cited, in accordance with accepted academic practice. No use, distribution or reproduction is permitted which does not comply with these terms. 